\title{
GENERALIZATIONS OF CLAUSEN'S FORMULA AND ALGEBRAIC TRANSFORMATIONS OF CALABI-YAU DIFFERENTIAL EQUATIONS
}

\author{
GERT ALMKVIST ${ }^{1}$, DUCO VAN STRATEN ${ }^{2}$ AND WADIM ZUDILIN ${ }^{3}$ \\ ${ }^{1}$ Matematikcentrum, Lunds Universitet, Matematik MNF, \\ Box 118, 22100 Lund, Sweden (gert@maths.lth.se) \\ ${ }^{2}$ Fachbereich Mathematik 08, Institut für Mathematik, AG Algebraische Geometrie, \\ Johannes Gutenberg-Universität, 55099 Mainz, Germany \\ (straten@mathematik.uni-mainz.de) \\ ${ }^{3}$ School of Mathematical and Physical Sciences, The University of Newcastle, \\ Callaghan, NSW 2308, Australia (wadim.zudilin@newcastle.edu.au)
}

(Received 8 July 2009)

\begin{abstract}
We provide certain unusual generalizations of Clausen's and Orr's theorems for solutions of fourth-order and fifth-order generalized hypergeometric equations. As an application, we present several examples of algebraic transformations of Calabi-Yau differential equations.
\end{abstract}

Keywords: Calabi-Yau differential equation; generalized hypergeometric series; monodromy; algebraic transformation

2010 Mathematics subject classification: Primary 33C20; 34M35

Secondary 05A10; 05A19; 14D05; 14J32; 32Q25; 32S40

\section{Introduction}

In our study of Picard-Fuchs differential equations of Calabi-Yau type $[\mathbf{2}, \mathbf{3}]$ we discovered some curious relations between hypergeometric series

$$
{ }_{m} F_{m-1}\left(\begin{array}{c}
a_{1}, a_{2}, \ldots, a_{m} \\
b_{2}, \ldots, b_{m}
\end{array} \mid z\right)=\sum_{n=0}^{\infty} \frac{\left(a_{1}\right)_{n}\left(a_{2}\right)_{n} \cdots\left(a_{m}\right)_{n}}{\left(b_{2}\right)_{n} \cdots\left(b_{m}\right)_{n}} \frac{z^{n}}{n !}
$$

and their natural generalizations. Although our original motivation was the differential equations themselves, we are intrigued to see that many of our identities can be extended to a more general form, which does not use all the properties of the Calabi-Yau prototypes. In addition to the classical examples of such identities, like Clausen's Formula

$$
{ }_{2} F_{1}\left(\begin{array}{c}
a, b \\
a+b+\frac{1}{2}
\end{array} \mid z\right)^{2}={ }_{3} F_{2}\left(\begin{array}{c}
2 a, 2 b, a+b \\
a+b+\frac{1}{2}, 2 a+2 b
\end{array} \mid z\right)
$$


and Orr-type theorems in $[\mathbf{1 7}, \S 2.5]$, we have already indicated an example related to a Calabi-Yau equation in [2, Proposition 6]. The main aim of the present paper is to systemize our findings and present algebraic transformations of certain hypergeometric and related series in a general form. One of our general theorems (Theorem 5.5) relates two different Hadamard products of second- and third-order hypergeometric-like differential equations in a way which could be counted as a higher-order analogue of (1.2). Specializations to Calabi-Yau examples $[\mathbf{2}, \mathbf{3}]$ are discussed in some detail.

The paper is organized as follows. In $\S 2$ we review the notion of a Calabi-Yau differential equation, while in $\S 3$ we recall some 'standard' relations between Calabi-Yau differential equations of order 2 and 3 , and of order 4 and 5 ; these two sections may be regarded as an expanded introductory part. Section 4 is devoted to algebraic transformations of second- and third-order differential equations. In $\S 5$ we discuss transformations of higher-order equations with applications to Calabi-Yau examples. In $\S 6$ we indicate a natural formal invariant of fourth-order Calabi-Yau differential equations that can be used to verify whether two such equations are related by an algebraic transformation. In $\S 7$ we discuss our strategies to find and prove algebraic transformations for differential equations.

\section{Calabi-Yau differential equations}

Certain differential equations look better than others, at least arithmetically. To illustrate this principle, consider the differential equation

$$
\left(\theta^{2}-z\left(11 \theta^{2}+11 \theta+3\right)-z^{2}(\theta+1)^{2}\right) y=0, \quad \text { where } \theta=z \frac{\mathrm{d}}{\mathrm{d} z} .
$$

What is special about it? First of all, it has a unique analytic solution $y_{0}(z)=f(z)$ with $f(0)=1$; another solution may be given in the form $y_{1}(z)=f(z) \log z+g(z)$ with $g(0)=0$. Secondly, the coefficients in the Taylor expansion $f(z)=\sum_{n=0}^{\infty} A_{n} z^{n}$ are integral, $f(z) \in 1+z \mathbb{Z} \llbracket z \rrbracket$, which can hardly be seen from the defining recurrence

$$
(n+1)^{2} A_{n+1}-\left(11 n^{2}+11 n+3\right) A_{n}-n^{2} A_{n-1}=0 \text { for } n=0,1, \ldots, A_{0}=1
$$

(cf. (2.1)), but follows from the explicit expression

$$
A_{n}=\sum_{k=0}^{n}\left(\begin{array}{l}
n \\
k
\end{array}\right)^{2}\left(\begin{array}{c}
n+k \\
n
\end{array}\right), \quad n=0,1, \ldots,
$$

due to Apéry [5]; note that these numbers appear in Apéry's proof of the irrationality of $\zeta(2)$. Thirdly, the expansion $q(z)=\exp \left(y_{1}(z) / y_{0}(z)\right)=z \exp (g(z) / f(z))$ also has integral coefficients, $q(z) \in z \mathbb{Z} \llbracket z \rrbracket$. This follows from the fact that the functional inverse $z(q)$,

$$
z(q)=q \prod_{n=1}^{\infty}\left(1-q^{n}\right)^{5(n / 5)}
$$

where $(n / 5)$ denotes the Legendre symbol, lies in $q \mathbb{Z} \llbracket q \rrbracket$. The formula in (2.4), due to Beukers $[7]$, shows that $z(q)$ is a modular function with respect to the congruence subgroup $\Gamma_{1}(5)$ of $\mathrm{SL}_{2}(\mathbb{Z})$. 
If the reader is not greatly surprised by these integrality properties, then we suggest trying to find more such cases, replacing the differential operator in (2.1) by the more general one

$$
\theta^{2}-z\left(a \theta^{2}+a \theta+b\right)+c z^{2}(\theta+1)^{2}
$$

To ensure the required integrality, one easily gets $a, b, c \in \mathbb{Z}$, but for a generic choice of the parameters the second feature $\left(y_{0}(z)=f(z) \in 1+z \mathbb{Z} \llbracket z \rrbracket\right)$ fails almost always. In fact, this problem was studied by Beukers $[\mathbf{8}]$ and Zagier $[\mathbf{2 0}]$. The exhaustive experimental search in $[\mathbf{2 0}]$ resulted in 14 (non-degenerate) examples of the triplets $(a, b, c) \in \mathbb{Z}^{3}$ when both this and the third property (the integrality of the corresponding expansion $z(q)$ ) hold; the latter follows from modular interpretations of $z(q)$.

A natural extension of the above problem to third-order linear differential equations is prompted by the other Apéry sequence used in his proof [5] of the irrationality of $\zeta(3)$. One takes the family of differential operators

$$
\theta^{3}-z(2 \theta+1)\left(\hat{a} \theta^{2}+\hat{a} \theta+\hat{b}\right)+\hat{c} z^{2}(\theta+1)^{3}
$$

and looks for the cases when the two solutions $f(z) \in 1+z \mathbb{C} \llbracket z \rrbracket$ and $f(z) \log z+g(z)$ (with $g(0)=0$ ) of the corresponding differential equation satisfy $f(z) \in \mathbb{Z} \llbracket z \rrbracket$ and $\exp (g(z) / f(z)) \in \mathbb{Z} \llbracket z \rrbracket$. Apart from some degenerate cases, we have again found in [2] 14 triplets $(\hat{a}, \hat{b}, \hat{c}) \in \mathbb{Z}^{3}$ meeting the integrality conditions; the second condition holds in all these cases as a modular bonus. Apéry's example corresponds to the case $(\hat{a}, \hat{b}, \hat{c})=$ $(17,5,1)$.

How can one generalize the above problem of finding 'arithmetically nice' linear differential equations (operators)? An approach we followed in $[\mathbf{2 , 3}]$, at least up to order 5 , was not specifying the form of the operator, as in (2.5) and (2.6), but rather imposing the following:

(i) the differential equation is of Fuchsian type, that is, all its singular points are regular; in addition, the local exponents at $z=0$ are zero;

(ii) the unique analytic solution $y_{0}(z)=f(z)$ with $f(0)=1$ at the origin has integral coefficients $f(z) \in 1+z \mathbb{Z} \llbracket z \rrbracket ;$ and

(iii) the solution $y_{1}(z)=f(z) \log z+g(z)$ with $g(0)=0$ gives rise to the integral expansion $\exp \left(y_{1}(z) / y_{0}(z)\right) \in z \mathbb{Z} \llbracket z \rrbracket$.

Requirement (i), known as the condition of maximally unipotent monodromy (MUM), means that the corresponding differential operator written as a polynomial in variable $z$ with coefficients from $\mathbb{C} \llbracket \theta \rrbracket$ has constant term $\theta^{m}$, where $m$ is the order (that is, the degree in $\theta$ ); the local monodromy around 0 consists of a single Jordan block of maximal size. Note that (i) guarantees the uniqueness of the above $y_{0}(z)$ and $y_{1}(z)$. Condition (ii) can be usually relaxed to $f(C z) \in 1+z \mathbb{Z} \llbracket z \rrbracket$ for some positive integer $C$ (without the scaling $z \mapsto C z$, many of the resulting formulae look 'more natural'). 
In fact, in $[\mathbf{2}, \mathbf{3}]$ we also imposed on fourth-order (and fifth-order) differential equations some extra conditions:

(iv) the 'Calabi-Yau' or 'self-duality' condition (3.4), which characterizes the structure of the projective monodromy group; and

(v) the integrality of a related sequence of numbers, known as instanton numbers in the physics literature; these arise as coefficients in the Lambert expansion of the so-called Yukawa coupling, which we review in $\S 6$.

For a long time we were confident that in all examples these additional conditions were satisfied automatically when (i)-(iii) hold. However, we have learnt recently from M. Bogner and S. Reiter (personal communication, March 2010) that the differential operator

$$
\theta^{4}-8 z(2 \theta+1)^{2}\left(5 \theta^{2}+5 \theta+2\right)+192 z^{2}(2 \theta+1)(2 \theta+3)(3 \theta+2)(3 \theta+4)
$$

satisfies conditions (i)-(iv) while condition (v) seems to fail.

Our experimental search $[\mathbf{2}, \mathbf{3}]$ resulted in more than 350 examples of such operators satisfying (i)-(v), which we called differential operators of Calabi-Yau type, since some of these examples can be identified with Picard-Fuchs differential equations for the periods of one-parameter families of Calabi-Yau manifolds. For an entry in our table from $[\mathbf{3}]$, checking (i) and (iv) is trivial, (ii) usually follows from an explicit form of the coefficients of $f(z)$ (when it is available), while (iii) can be verified in certain cases using some of Dwork's $p$-adic techniques. Substantial progress in this direction was obtained recently by Krattenthaler and Rivoal [11]. According to standard conjectures (see, for example, [4]) all our operators should be of geometric origin, meaning that they correspond (as subquotients of the local systems) to factors of Picard-Fuchs equations satisfied by period integrals for some family of varieties over the projective line. Finally, a rigorous verification of condition ( $\mathrm{v}$ ) remains beyond the reach of available methods; we only have computational evidence for integrality of the instanton numbers.

Basic examples of Calabi-Yau differential equations are given by the general hypergeometric differential equation

$$
\left(\theta \prod_{j=2}^{m}\left(\theta+b_{j}-1\right)-z \prod_{j=1}^{m}\left(\theta+a_{j}\right)\right) y=0
$$

of order $m$ satisfied by the hypergeometric series (1.1). The equation (2.8) has (smallest possible) degree 1 in $z$ and condition (i) forces $b_{2}=\cdots=b_{m}=1$ to hold. The latter is the main reason for our identities below to involve the hypergeometric series with this special form of the lower parameters.

\section{Symmetric and antisymmetric squares}

Given a second-order linear homogeneous differential equation

$$
y^{\prime \prime}+P y^{\prime}+Q y=0
$$


(where the prime denotes $\mathrm{d} / \mathrm{d} z$ ) and a pair of its two linearly independent solutions $y_{0}=y_{0}(z)$ and $y_{1}=y_{1}(z)$, one can easily construct the third-order differential equation whose solutions are $y_{0}^{2}, y_{0} y_{1}$ and $y_{1}^{2}$ :

$$
y^{\prime \prime \prime}+3 P y^{\prime \prime}+\left(2 P^{2}+P^{\prime}+4 Q\right) y^{\prime}+\left(4 P Q+2 Q^{\prime}\right) y=0,
$$

called the symmetric square of (3.1) (see [19, Chapter 14, Exercise 10]). Clearly, (3.2) is independent of a choice of solutions $y_{0}, y_{1}$ of the equation (3.1). A hypergeometric example of the relationship between solutions of (3.1) and (3.2) is Clausen's Formula (1.2).

The situation changes drastically when one goes to linear homogeneous differential equations of order higher than 2. In principle, there is no difficulty in writing formulae similar to (3.2) for the symmetric cubes, biquadratics, etc, but unfortunately, as far as we know, this never results in some non-trivial identities for the hypergeometric series (1.1).

If the coefficients of a fourth-order linear differential equation

$$
y^{(\mathrm{iv})}+P y^{\prime \prime \prime}+Q y^{\prime \prime}+R y^{\prime}+S y=0
$$

satisfy the relation

$$
R=\frac{1}{2} P Q-\frac{1}{8} P^{3}+Q^{\prime}-\frac{3}{4} P P^{\prime}-\frac{1}{2} P^{\prime \prime},
$$

then the equation and the corresponding operator are said to satisfy the Calabi-Yau condition [2]; the equation expresses the self-duality of (3.3). To avoid possible confusion, (3.4) is only one condition characterizing the class of Calabi-Yau differential equations and operators; the latter should satisfy conditions (i)-(v) of $\S 2$. However, our theorems below refer to linear differential equations of more general type, not just to Calabi-Yau examples.

If $y_{0}, y_{1}, y_{2}, y_{3}$ are linearly independent solutions, then condition (3.4) implies that the six functions

$$
w_{j k}=W\left(y_{j}, y_{k}\right)=\operatorname{det}\left(\begin{array}{ll}
y_{j} & y_{k} \\
y_{j}^{\prime} & y_{k}^{\prime}
\end{array}\right), \quad 0 \leqslant j<k \leqslant 3,
$$

are linearly dependent over $\mathbb{C}$. These functions satisfy a fifth-order linear differential equation

$$
y^{(\mathrm{v})}+\tilde{P} y^{(\mathrm{iv})}+\tilde{Q} y^{\prime \prime \prime}+\tilde{R} y^{\prime \prime}+\tilde{S} y^{\prime}+\tilde{T} y=0,
$$

independent of the choice of solutions $y_{0}, y_{1}, y_{2}, y_{3}$ of (3.4) and called the antisymmetric square of (3.4) (see, for example, [2, Proposition 1]).

Proposition 3.1 (Almkvist [1]; Y. Yang (personal communication, November 2006)). Suppose that a fifth-order equation (3.6) is the antisymmetric square of a fourthorder linear differential equation. Let $U=U(z)$ be an arbitrary function. Then, for any pair $w_{0}, w_{1}$ of solutions of (3.6), the function

$$
y=W\left(w_{0}, w_{1}\right)^{1 / 2} \cdot U
$$

satisfies a fourth-order equation (3.4) whose coefficients $P, Q, R$ and $S$ are differential polynomials in $\tilde{P}, \tilde{Q}, \tilde{R}, \tilde{S}, \tilde{T}$ and $U$. (The explicit expressions are given in [1].) 
Following $[\mathbf{1}]$ we call the resulting fourth-order equation (3.4) with the choice

$$
U=z^{5 / 2} \exp \left\{-\frac{1}{5} \int^{z} \tilde{P}(z) \mathrm{d} z\right\}
$$

the Yifan Yang pullback of (3.6), or the YY-pullback for short. Note that for Calabi-Yau equations the exponential factor in (3.8) is an algebraic expression.

As an example, the YY-pullback of the equation

$$
\left(\theta^{5}-z\left(\theta+\frac{1}{2}\right)(\theta+\alpha)(\theta+1-\alpha)(\theta+\beta)(\theta+1-\beta)\right) y=0,
$$

satisfied by the hypergeometric function

$$
{ }_{5} F_{4}\left(\begin{array}{c|c}
\frac{1}{2}, \alpha, 1-\alpha, \beta, 1-\beta & z \\
1,1,1,1 & \mid
\end{array}\right)
$$

is given $[\mathbf{1}]$ by

$$
\begin{aligned}
& \left(\theta^{4}-z\left(2\left(\theta+\frac{1}{2}\right)^{4}+\frac{1}{2}\left(\theta+\frac{1}{2}\right)^{2}(\alpha(1-\alpha)+\beta(1-\beta)+3)\right.\right. \\
& \left.-\frac{1}{4} \alpha(1-\alpha) \beta(1-\beta)+\frac{1}{8} \alpha(1-\alpha)+\frac{1}{8} \beta(1-\beta)\right) \\
& +z^{2}\left(\theta+\frac{1}{2}+\frac{1}{2}(\alpha+\beta)\right)\left(\theta+\frac{1}{2}+\frac{1}{2}(\alpha+1-\beta)\right)\left(\theta+\frac{1}{2}+\frac{1}{2}(1-\alpha+\beta)\right) \\
& \left.\times\left(\theta+\frac{1}{2}+\frac{1}{2}(1-\alpha+1-\beta)\right)\right) y=0 .
\end{aligned}
$$

As we will see in the theorems of $\S 5$, many Calabi-Yau differential equations related by algebraic transformations are Hadamard products of second- and third-order PicardFuchs differential equations, with 0 a MUM point. Recall that the Hadamard product of two series

$$
f(z)=\sum_{n=0}^{\infty} A_{n} z^{n} \quad \text { and } \quad \hat{f}(z)=\sum_{n=0}^{\infty} \hat{A}_{n} z^{n}
$$

is defined by the formula

$$
f(z) * \hat{f}(z)=\sum_{n=0}^{\infty} A_{n} \hat{A}_{n} z^{n} .
$$

If $f(z)$ and $\hat{f}(z)$ are the analytic solutions of two differential equations $D y=0$ and $\hat{D} y=0$, respectively, then their Hadamard product $f(z) * \hat{f}(z)$ satisfies a differential equation $\tilde{D} y=0$ (we pick the one of minimal order), which we then call the Hadamard product of the two equations. The differential operator $\tilde{D}$ in this case is the Hadamard product of the corresponding operators $D$ and $\hat{D}$. The Hadamard product is the analytic representation of the multiplicative convolution. In particular, the singular points of the operator $\tilde{D}$ consist of the products of singular points of $D$ and of $\hat{D}$. The Hadamard product of operators of geometric origin is again of geometric origin (see, for example, [4]).

We will say that two (Calabi-Yau) differential equations or operators are equivalent if they are related by an algebraic transformation. Note that algebraic transformations preserve the order of differential equations with rational or algebraic coefficients. 


\section{Apéry-like differential operators}

To illustrate the above theorems and also to present some further algebraic transformations, we will list second- and third-order Calabi-Yau equations, keeping the names used in $[\mathbf{2}, \mathbf{3}, \mathbf{1 8}]$.

In writing down the series for analytic solutions of the Calabi-Yau differential equations, one usually re-normalizes the variable $z \mapsto C z$ in order to make the series expansions lying in $1+z \mathbb{Z} \llbracket z \rrbracket$ (see condition (ii) in $\S 2$ ). Basic examples are ${ }_{2} F_{1}$-hypergeometric series satisfying second-order differential equations, and there are exactly four such series having MUM at the origin (that is, satisfying condition (i)):

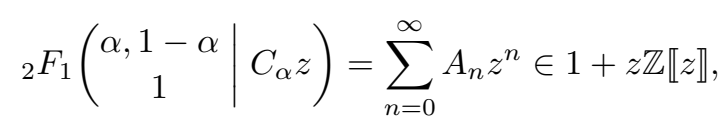

where

$$
\left.\begin{array}{ll}
\text { (A) } \quad \alpha=\frac{1}{2}, & C_{1 / 2}=16=2^{4}, \\
\text { (B) } \quad \alpha=\frac{1}{3}, & C_{1 / 3}=27=3^{3}, \\
\text { (C) } \quad \alpha=\frac{1}{4}, & C_{1 / 4}=64=2^{6}, \\
\text { (D) } \quad \alpha=\frac{1}{6}, & C_{1 / 6}=432=2^{4} \cdot 3^{3} .
\end{array}\right\}
$$

The corresponding differential operators are

$$
\theta^{2}-C_{\alpha} z(\theta+\alpha)(\theta+1-\alpha)
$$

and the integrality of the expansions in (4.1) follows from the explicit formulae

$$
\left.\begin{array}{l}
\text { (A) } A_{n}=\left(\begin{array}{c}
2 n \\
n
\end{array}\right)^{2}, \\
\text { (B) } A_{n}=\frac{(3 n) !}{n !^{3}} \\
\text { (C) } A_{n}=\frac{(4 n) !}{n !^{2}(2 n) !} \\
\text { (D) } A_{n}=\frac{(6 n) !}{n !(2 n) !(3 n) !} .
\end{array}\right\}
$$

These four hypergeometric instances are particular examples of the second-order differential operators having the form (2.5); they correspond to the choice $c=0$. In addition to the above hypergeometric cases, Zagier [20] found four Legendrian and six sporadic equations.

The Legendrian examples are obtained from the hypergeometric ones by a simple rational transformation that interchanges $1 / C_{\alpha}$ and $\infty$ :

$$
\frac{1}{1-C_{\alpha} z} \cdot{ }_{2} F_{1}\left(\begin{array}{c|c}
\alpha, 1-\alpha & \frac{-C_{\alpha} z}{1-C_{\alpha} z}
\end{array}\right) \in 1+z \mathbb{Z} \llbracket z \rrbracket
$$


where

$$
\begin{array}{llll}
\text { (e) } \alpha=\frac{1}{2}, & \text { (h) } \alpha=\frac{1}{3}, & \text { (i) } \alpha=\frac{1}{4}, & \text { (j) } \alpha=\frac{1}{6} \text {; }
\end{array}
$$

the corresponding differential operators

$$
\theta^{2}-C_{\alpha} z\left(\theta^{2}+(\theta+1)^{2}-\alpha(1-\alpha)\right)+C_{\alpha}^{2} z^{2}(\theta+1)^{2}
$$

have the form (2.5) with $c=a^{2} / 4$.

These four hypergeometric operators and their Legendrian companions have a nice geometric origin: they are Picard-Fuchs operators of the extremal rational elliptic surfaces with three singular fibres $[\mathbf{1 3}, \mathbf{1 6}]$.

The sporadic examples of (2.5) (when $c \neq 0$ and $c \neq a^{2} / 4$ as in the hypergeometric and Legendrian cases, respectively) with the corresponding analytic solutions $\sum_{n=0}^{\infty} A_{n} z^{n} \in$ $1+z \mathbb{Z} \llbracket z \rrbracket$ are as follows:
(a) $\quad a=7, \quad b=2, \quad c=-8$,
$A_{n}=\sum_{k}\left(\begin{array}{l}n \\ k\end{array}\right)^{3}$
(b) $\quad a=11, \quad b=3, \quad c=-1$,$$
A_{n}=\sum_{k}\left(\begin{array}{l}
n \\
k
\end{array}\right)^{2}\left(\begin{array}{c}
n+k \\
n
\end{array}\right)
$$
(c) $\quad a=10, \quad b=3, \quad c=9$,$$
A_{n}=\sum_{k}\left(\begin{array}{l}
n \\
k
\end{array}\right)^{2}\left(\begin{array}{c}
2 k \\
k
\end{array}\right)
$$
(d) $\quad a=12, \quad b=4, \quad c=32$,$$
A_{n}=\sum_{k}\left(\begin{array}{c}
n \\
k
\end{array}\right)\left(\begin{array}{c}
2 k \\
k
\end{array}\right)\left(\begin{array}{c}
2 n-2 k \\
n-k
\end{array}\right)
$$
(f) $\quad a=9, \quad b=3, \quad c=27$,$$
A_{n}=\sum_{k}(-1)^{k} 3^{n-3 k}\left(\begin{array}{c}
n \\
3 k
\end{array}\right) \frac{(3 k) !}{k !^{3}}
$$
(g) $\quad a=17, \quad b=6, \quad c=72$,$$
A_{n}=\sum_{k, l}(-1)^{k} 8^{n-k}\left(\begin{array}{l}
n \\
k
\end{array}\right)\left(\begin{array}{l}
k \\
l
\end{array}\right)^{3} .
$$

These six sporadic operators also have a geometric origin; they arise as Picard-Fuchs equations of the six families of elliptic curves with four reduced singular fibres $[\mathbf{6}, \mathbf{1 3}$, although the connection between the operators and rational elliptic surfaces is not oneto-one (cf. [20]).

The story for the third-order differential operators of the form (2.6) looks very similar to that for order 2. We also have four hypergeometric examples

$$
{ }_{3} F_{2}\left(\begin{array}{c|c}
\frac{1}{2}, \alpha, 1-\alpha \\
1,1
\end{array} \mid 4 C_{\alpha} z\right) \in 1+z \mathbb{Z} \llbracket z \rrbracket
$$

four operators of Legendre type and six sporadic operators. 
The 'Legendrian' third-order examples originate from the series

$$
\begin{aligned}
\sum_{n=0}^{\infty} A_{n} z^{n} & =\sum_{n=0}^{\infty}\left(C_{\alpha} z\right)^{n} \sum_{k=0}^{n}\left(\frac{(\alpha)_{k}(1-\alpha)_{n-k}}{k !(n-k) !}\right)^{2} \\
& =\frac{1}{1-C_{\alpha} z}{ }^{3} F_{2}\left(\begin{array}{c|c}
\frac{1}{2}, \alpha, 1-\alpha & -4 C_{\alpha} z \\
1,1 & \frac{\left.1-C_{\alpha} z\right)^{2}}{\left(1-C_{\alpha}\right.}
\end{array}\right)^{2} \\
& =\frac{1}{1-C_{\alpha} z}{ }_{2} F_{1}\left(\begin{array}{c}
\alpha, 1-\alpha \\
1
\end{array} \mid \frac{-C_{\alpha} z}{1-C_{\alpha}}\right.
\end{aligned}
$$

(we use $[\mathbf{1 7}, \S 2.5$, Theorem IX] and the Euler transformation $[\mathbf{1 7}$, p. 31, (1.7.1.3)]), where

$$
(\beta) \alpha=\frac{1}{2}, \quad(\iota) \alpha=\frac{1}{3}, \quad(\vartheta) \alpha=\frac{1}{4}, \quad(\kappa) \alpha=\frac{1}{6} ;
$$

the corresponding differential operators are

$$
\theta^{3}-C_{\alpha} z(2 \theta+1)\left(\theta(\theta+1)+\alpha^{2}+(1-\alpha)^{2}\right)+C_{\alpha}^{2} z^{2}(\theta+1)^{3} .
$$

The sporadic third-order examples of (2.6) with analytic solutions

$$
\sum_{n=0}^{\infty} A_{n} z^{n} \in 1+z \mathbb{Z} \llbracket z \rrbracket
$$

are given in the following list:

$$
\begin{array}{lll}
(\delta) \hat{a}=7, & \hat{b}=3, \quad \hat{c}=81, & A_{n}=\sum_{k}(-1)^{k} 3^{n-3 k}\left(\begin{array}{c}
n \\
3 k
\end{array}\right)\left(\begin{array}{c}
n+k \\
n
\end{array}\right) \frac{(3 k) !}{k !^{3}} \\
(\eta) \hat{a}=11, & \hat{b}=5, \quad \hat{c}=125, & A_{n}=\sum_{k}(-1)^{k}\left(\begin{array}{l}
n \\
k
\end{array}\right)^{3}\left(\left(\begin{array}{c}
4 n-5 k-1 \\
3 n
\end{array}\right)+\left(\begin{array}{c}
4 n-5 k \\
3 n
\end{array}\right)\right) \\
(\alpha) \hat{a}=10, \quad \hat{b}=4, \quad \hat{c}=64, & A_{n}=\sum_{k}\left(\begin{array}{l}
n \\
k
\end{array}\right)^{2}\left(\begin{array}{c}
2 k \\
k
\end{array}\right)\left(\begin{array}{c}
2 n-2 k \\
n-k
\end{array}\right) \\
(\epsilon) \hat{a}=12, & \hat{b}=4, \quad \hat{c}=16, & A_{n}=\sum_{k}\left(\begin{array}{l}
n \\
k
\end{array}\right)^{2}\left(\begin{array}{c}
2 k \\
n
\end{array}\right)^{2} \\
(\zeta) \hat{a}=9, \quad \hat{b}=3, \quad \hat{c}=-27, & A_{n}=\sum_{k, l}\left(\begin{array}{l}
n \\
k
\end{array}\right)^{2}\left(\begin{array}{c}
n \\
l
\end{array}\right)\left(\begin{array}{c}
k \\
l
\end{array}\right)\left(\begin{array}{c}
k+l \\
n
\end{array}\right) \\
(\gamma) \hat{a}=17, \quad \hat{b}=5, \quad \hat{c}=1, & A_{n}=\sum_{k}\left(\begin{array}{c}
n \\
k
\end{array}\right)^{2}\left(\begin{array}{c}
n+k \\
n
\end{array}\right)^{2} .
\end{array}
$$

The following theorem gives a natural bijection between the differential operators (2.5) and (2.6), in particular, between the above 14 pairs of arithmetic operators.

Theorem 4.1. Let the triplets $(a, b, c)$ and $(\hat{a}, \hat{b}, \hat{c})$ be related by the formulae

$$
\hat{a}=a, \quad \hat{b}=a-2 b \quad \text { and } \quad \hat{c}=a^{2}-4 c .
$$


For the differential operators $D$ and $\hat{D}$ given in (2.5) and (2.6), denote by $f(z)$ and $\hat{f}(z)$ the analytic solutions of $D y=0$ and $\hat{D} y=0$, respectively, with $f(0)=\hat{f}(0)=1$. Then

$$
f(z)^{2}=\frac{1}{1-a z+c z^{2}} \hat{f}\left(\frac{-z}{1-a z+c z^{2}}\right) .
$$

Proof. Writing down the general third-order differential equations for the functions on the left- and right-hand sides of (4.14), respectively, is a routine exercise in MAPLE to show that the transformation is the right one.

In fact, there is a natural geometric construction that explains this bijection, which we will sketch now. The second-order operators are Picard-Fuchs operators for special families of elliptic curves $E_{t}$, where $t \in Y=\mathbb{P}^{1}$. In each of the cases the rational curve $Y$ covers the modular curve $X_{0}(N)$ for some $N$, so that each elliptic curve $E_{t}$ comes with a cyclic subgroup of order $N$. The quotient of $E_{t}$ by this cyclic subgroup turns out to be $E_{\iota(t)}$, where $\iota: Y \rightarrow Y$ is an involution corresponding to the Atkin-Lehner involution that acts as $\tau \mapsto-1 /(N \tau)$ on the elliptic modular parameter. The product of the elliptic curves $A_{t}:=E_{t} \times E_{\iota(t)}$ can now be considered as parametrized by the rational curve $Z:=Y / \iota$. The Picard-Fuchs equation for the holomorphic 2-form for this family is of order 3 and thus can be seen as a 'twisted' square of the corresponding second-order operators. We refer the reader to $[\mathbf{1 0}, \mathbf{1 4}, \mathbf{1 5}]$ for details about this construction.

The specific form of the transformation can be understood by noting that the quotient map $Y \rightarrow Z$ is described by a degree-2 rational map $f: \mathbb{P}^{1} \rightarrow \mathbb{P}^{1}$. If the pre-image of 0 consists of the points 0 and $\infty$, and the pre-image of $\infty$ of the two other singular points of the second-order operator (2.5) (that is, of the roots of $1-a z+c z^{2}$ ), then one is led to a map of the form $z \mapsto e z /\left(1-a z+c z^{2}\right)$. The singular points of the third-order operator (2.6) consist of $0, \infty$ and the roots of the equation $1-2 \hat{a} z+\hat{c} z^{2}=0$, and these have to coincide with the image of the two critical points of the map, which one computes to be the roots of $e^{2}+2 a e z+z^{2}\left(a^{2}-4 c\right)$. Hence, one can take $e=-1, \hat{a}=a$ and $\hat{c}=a^{2}-4 c$. The factor in front of $\hat{f}$ in (4.14) is needed to get the local exponents agreed, but the value of $\hat{b}$ remains undetermined by these considerations.

\section{Hadamard products and algebraic transformations}

Recall that the YY-pullback of the differential equation (3.9) is (3.11). The latter fourthorder equation has a unique analytic solution of the form

$$
\tilde{F}(z) \in 1+z \mathbb{C} \llbracket z \rrbracket
$$

at the origin, since all exponents at $z=0$ are zero (in other words, (3.11) has MUM at the origin). Roughly speaking, we may call the function $\tilde{F}(z)$ an antisymmetric square root of (3.10). The following theorem may be viewed as a generalization of Clausen's Formula (1.2) in the special case $a+b=\frac{1}{2}$. 
Theorem 5.1. Let

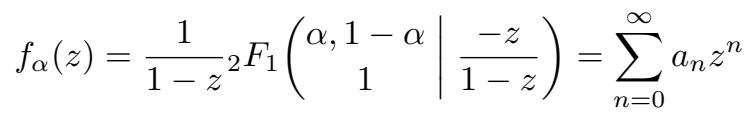

and

$$
f_{\beta}(z)=\frac{1}{1-z}{ }_{2} F_{1}\left(\begin{array}{c|c}
\beta, 1-\beta & \frac{-z}{1-z}
\end{array}\right)=\sum_{n=0}^{\infty} b_{n} z^{n},
$$

and let $F(z)$ be the Hadamard product of the series $f_{\alpha}(z)$ and $f_{\beta}(z)$,

$$
F(z)=\sum_{n=0}^{\infty} a_{n} b_{n} z^{n}
$$

Then for the analytic solution (5.1) of the YY-pullback (3.11) of (3.9) we have

$$
F(z)=\frac{1+z / 4}{(1-z / 4)^{2}} \tilde{F}\left(\frac{-z / 4}{(1-z / 4)^{2}}\right)
$$

equivalently,

$$
\tilde{F}(z)=\frac{2}{1-z+\sqrt{1-z}} F\left(\frac{-z / 2}{1-z / 2+\sqrt{1-z}}\right) .
$$

Remark 5.2. The coefficients $a_{n}$ in the expansion (5.2) may be given by the formulae

$$
a_{n}=\sum_{k=0}^{n}(-1)^{k}\left(\begin{array}{l}
n \\
k
\end{array}\right) \frac{(\alpha)_{k}(1-\alpha)_{k}}{k !^{2}}=\sum_{k=0}^{n} \frac{(\alpha)_{k}}{k !} \frac{(1-\alpha)_{n-k}^{2}}{(n-k) !^{2}}
$$

similar formulae, but with the substitution of $\beta$ for $\alpha$, are available for the coefficients $b_{n}$. The statement of Theorem 5.1 is a version of the experimental observation in $[\mathbf{1}, \S 3.2]$. Recalling the relationship between solutions of (3.9) and (3.11), we can write our final formula (5.6) as follows (a little reminiscent of Clausen's original formula (1.2)):

$$
\begin{gathered}
\sum_{n=0}^{\infty} z^{n} \sum_{k=0}^{n} \frac{\left(\frac{1}{2}\right)_{k}\left(\frac{1}{2}\right)_{n-k}(\alpha)_{k}(\alpha)_{n-k}(1-\alpha)_{k}(1-\alpha)_{n-k}(\beta)_{k}(\beta)_{n-k}(1-\beta)_{k}(1-\beta)_{n-k}}{k !^{5}(n-k) !^{5}} \\
\times\left(1+(2 k-n) \sum_{j=0}^{k-1}\left(\frac{1}{\frac{1}{2}+j}+\frac{1}{\alpha+j}+\frac{1}{1-\alpha+j}+\frac{1}{\beta+j}+\frac{1}{1-\beta+j}\right)\right) \\
=\frac{4(1-z)}{(1-z+\sqrt{1-z})^{2}}\left(\sum_{n=0}^{\infty}\left(\frac{-z / 2}{1-z / 2+\sqrt{1-z}}\right)^{n}\right. \\
\left.\times \sum_{j=0}^{n}(-1)^{j}\left(\begin{array}{l}
n \\
j
\end{array}\right) \frac{(\alpha)_{j}(1-\alpha)_{j}}{j !^{2}} \cdot \sum_{k=0}^{n}(-1)^{k}\left(\begin{array}{l}
n \\
k
\end{array}\right) \frac{(\beta)_{k}(1-\beta)_{k}}{k !^{2}}\right)^{2} .
\end{gathered}
$$

Proof of Theorem 5.1. The sequence (5.7) satisfies the recursion

$$
(n+1)^{2} a_{n+1}-\left(n^{2}+(n+1)^{2}-\alpha(1-\alpha)\right) a_{n}+n^{2} a_{n-1}=0,
$$


and a similar formula is valid for the sequence $b_{n}$. Taking the Hadamard product $a_{n} b_{n}$ in $(5.4)$ as described in $[\mathbf{2}, \S 7]$ gives a fourth-order recursion (which is too plain to be stated here). The corresponding differential operator $\mathcal{L}$ annihilating the series (5.4) is of order 6 and is factorizable, $\mathcal{L}=\mathcal{L}_{1} \mathcal{L}_{2}$, where $\mathcal{L}_{1}$ is of order 2. Computing $\mathcal{L}_{1}$ and performing leftdivision $\left(\mathcal{L}, \mathcal{L}_{1},[\mathrm{~d} / \mathrm{d} z, z]\right)$ in MAPLE, we find $\mathcal{L}_{2}$, which can be written in the form

$$
\begin{aligned}
\mathcal{L}_{2}=\theta^{4} & -z\left(2 \theta^{4}+8 \theta^{3}-2(s-4) \theta^{2}-2(s-2) \theta+p-s+1\right) \\
& -z^{2}\left(\theta^{4}-12 \theta^{3}-26 \theta^{2}+4(s-5) \theta+4 p-s^{2}+4 s-7\right) \\
& +z^{3}\left(4 \theta^{4}+8 \theta^{3}-4(s+3) \theta^{2}-4(s+4) \theta-6 p+2 s^{2}+2 s-8\right) \\
& -z^{4}\left(\theta^{4}+16 \theta^{3}+16 \theta^{2}-4(s-2) \theta+4 p-s^{2}\right) \\
& -z^{5}\left(2 \theta^{4}-2(s+2) \theta^{2}-2(s+2) \theta+p-s-1\right)+z^{6}(\theta+1)^{4},
\end{aligned}
$$

where $s=\alpha(1-\alpha)+\beta(1-\beta)$ and $p=\alpha(1-\alpha) \beta(1-\beta)$. Then we finish the proof by performing the transformation

$$
z=\frac{-4 Z}{(1-Z)^{2}}, \quad y(z)=\frac{(1-Z)^{2}}{2(1+Z)} Y(Z)
$$

which transforms the equation for $\tilde{F}(z)$ to $\mathcal{L}_{2} Y=0$. (Some precaution is necessary, since MAPLE does not cancel common factors in the coefficients of the resulting differential equation.) This equation has the unique analytic solution at the origin and both expansions in (5.6) lie in $1+z \mathbb{C} \llbracket z \rrbracket$.

In the cases $\alpha, \beta \in\left\{\frac{1}{2}, \frac{1}{3}, \frac{1}{4}, \frac{1}{6}\right\}$, Theorem 5.1 provides the equivalences for the YY-pullbacks of fifth-order Calabi-Yau hypergeometric differential equations and the fourth-order Hadamard products of Legendrian cases (4.5)-(4.7).

Our next family of transformations concerns the series

$$
\begin{aligned}
g_{\alpha}(z) & ={ }_{2} F_{1}\left(\begin{array}{c|c}
\alpha, \alpha & z \\
1 & z
\end{array}\right) \cdot{ }_{2} F_{1}\left(\begin{array}{c}
1-\alpha, 1-\alpha \\
1
\end{array} \mid z\right) \\
& =\frac{1}{1-z}{ }_{3} F_{2}\left(\begin{array}{c}
\frac{1}{2}, \alpha, 1-\alpha \\
1,1
\end{array} \mid \frac{-4 z}{(1-z)^{2}}\right) ;
\end{aligned}
$$

the particular cases correspond to the Legendrian third-order examples from $\S 4$. Writing $g_{\alpha}(z)=\sum_{n=0}^{\infty} a_{n} z^{n}$ and using the first representation in (5.9), one finds that

$$
a_{n}=\sum_{k=0}^{n}\left(\frac{(\alpha)_{k}(1-\alpha)_{n-k}}{k !(n-k) !}\right)^{2},
$$

and this sequence satisfies the recursion

$$
(n+1)^{3} a_{n+1}-(2 n+1)\left(n(n+1)+\alpha^{2}+(1-\alpha)^{2}\right) a_{n}+n^{3} a_{n-1}=0 .
$$


Applying twice the Euler transformation [17, p. 31, Equation (1.7.1.3)] to the first expression in (5.9) gives one a way to express $g_{\alpha}(z)$ as the square:

$$
g_{\alpha}(z)=\frac{1}{1-z}{ }_{2} F_{1}\left(\begin{array}{c|c}
\alpha, 1-\alpha & -z \\
1 & 1-z
\end{array}\right)^{2}
$$

Theorem 5.3. Let $F(z)$ be the Hadamard product of ${ }_{2} F_{1}\left(\begin{array}{c}\alpha, 1-\alpha \\ 1\end{array} \mid z\right)$ and $f_{\beta}(z)$ in (5.3), and let $G(z)$ be the Hadamard product of ${ }_{2} F_{1}\left({ }_{1}^{\beta, 1-\beta} \mid z\right)$ and $g_{\alpha}(z)$ in (5.9), (5.12). Let $\tilde{G}(z) \in 1+z \mathbb{C} \llbracket z \rrbracket$ be the analytic solution of the $Y Y$-pullback

$$
\begin{aligned}
& \left(\theta^{4}-z\left(4\left(\theta+\frac{1}{2}\right)^{4}\right.\right. \\
& \left.\quad+(4-2 \alpha(1-\alpha)+\beta(1-\beta))\left(\theta+\frac{1}{2}\right)^{2}+\frac{1}{8}+\left(\alpha(1-\alpha)-\frac{1}{4}\right)\left(\beta(1-\beta)-\frac{1}{2}\right)\right) \\
& +z^{2}\left(6(\theta+1)^{4}+\left(\frac{15}{2}-4 \alpha(1-\alpha)+3 \beta(1-\beta)\right)(\theta+1)^{2}\right. \\
& \left.\quad \quad+\frac{3}{4}+\alpha(1-\alpha) \beta(1-\beta)+\alpha^{2}(1-\alpha)^{2}-\alpha(1-\alpha)\right) \\
& \quad-z^{3}\left(\theta+\frac{3}{2}\right)^{2}\left(4\left(\theta+\frac{3}{2}\right)^{2}+3-2 \alpha(1-\alpha)-3 \beta(1-\beta)\right) \\
& \left.+z^{4}\left(\theta+\frac{3}{2}\right)\left(\theta+\frac{5}{2}\right)\left(\theta+\beta+\frac{3}{2}\right)\left(\theta-\beta+\frac{5}{2}\right)\right) y=0
\end{aligned}
$$

of the fifth-order linear differential equation satisfied by $G(z)$. Then

$$
F(z)=\tilde{G}\left(\frac{-z}{1-z}\right) \quad \text { and } \quad \tilde{G}(z)=F\left(\frac{-z}{1-z}\right) .
$$

Proof. The proof is via a routine in the spirit of the proof of Theorem 5.1.

In $[\mathbf{2 1}]$ we consider a quadratic transformation of a ${ }_{5} F_{4}$-series with a particular instance

$$
{ }_{5} F_{4}\left(\begin{array}{c}
\frac{1}{2}, \frac{1}{2}, \frac{1}{2}, \frac{1}{2}, \frac{1}{2} \\
1,1,1,1
\end{array} \mid z\right)=\frac{1}{(1-z)^{1 / 2}} \sum_{n=0}^{\infty}\left(\frac{-4 z}{(1-z)^{2}}\right)^{n} \frac{\left(\frac{1}{4}\right)_{n}\left(\frac{3}{4}\right)_{n}}{n !^{2}} a_{n},
$$

where

$$
a_{n}=\sum_{k=0}^{n} \frac{\left(\frac{1}{2}\right)_{k}^{3}}{k !^{3}} \frac{\left(\frac{1}{2}\right)_{n-k}}{(n-k) !}=\sum_{k=0}^{n}\left(\frac{\left(\frac{1}{4}\right)_{k}\left(\frac{3}{4}\right)_{n-k}}{k !(n-k) !}\right)^{2}
$$

are coefficients in the power expansion of $g_{1 / 4}(z)$ in (5.9).

The series on the left-hand side in (5.15) is the special case $\alpha=\beta=\frac{1}{2}$ of (3.10), and Theorem 5.1 gives an example of quadratic transformation of the corresponding YYpullback (3.11). Our next theorem gives another quadratic transformation for the series $F(z)$, from (5.4) in this case.

Theorem 5.4. Set $f(z)=f_{1 / 2}(z)$ and $\hat{f}(z)=f_{1 / 4}(z)$, where $f_{\alpha}(z)$ is defined in (5.2). Let $F(z)$ be the Hadamard square of the series $f(z)$,

$$
F(z)=\sum_{n=0}^{\infty} z^{n}\left(\sum_{k=0}^{n}(-1)^{k}\left(\begin{array}{l}
n \\
k
\end{array}\right) \frac{\left(\frac{1}{2}\right)_{k}^{2}}{k !^{2}}\right)^{2}
$$


and let $\hat{F}(z)$ be the Hadamard product of ${ }_{2} F_{1}(\underset{1}{1 / 4,3 / 4} \mid z)$ and $\hat{f}(z)$,

$$
\hat{F}(z)=\sum_{n=0}^{\infty} z^{n} \frac{\left(\frac{1}{4}\right)_{n}\left(\frac{3}{4}\right)_{n}}{n !^{2}} \sum_{k=0}^{n}(-1)^{k}\left(\begin{array}{l}
n \\
k
\end{array}\right) \frac{\left(\frac{1}{4}\right)_{k}\left(\frac{3}{4}\right)_{k}}{k !^{2}} .
$$

Then

$$
F(z)=\frac{1}{\sqrt{1-6 z+z^{2}}} \hat{F}\left(\frac{-16 z(1-z)^{2}}{\left(1-6 z+z^{2}\right)^{2}}\right) .
$$

Proof. The proof is routine (cf. $\S 7$ ).

Our next result refers to a generic set of the (complex) parameters $\alpha, a, b$ and $c$, while the three additional parameters $\hat{a}, \hat{b}$ and $\hat{c}$ are defined in accordance with (4.13). The Hadamard product of the differential operators $\theta^{2}-z(\theta+\alpha)(\theta+1-\alpha)$ (which is the un-normalized version of (4.3)) and (2.6) is

$$
\begin{aligned}
\theta^{5}-z(2 \theta+1)(\theta+\alpha) & (\theta+1-\alpha)\left(\hat{a} \theta^{2}+\hat{a} \theta+\hat{b}\right) \\
& +\hat{c} z^{2}(\theta+1)(\theta+\alpha)(\theta+1-\alpha)(\theta+1+\alpha)(\theta+2-\alpha) ;
\end{aligned}
$$

its fourth-order YY-pullback reads

$$
\begin{aligned}
D=\theta^{4} & -z\left(4 \hat{a}\left(\theta+\frac{1}{2}\right)^{4}+((p+4) \hat{a}-2 \hat{b})\left(\theta+\frac{1}{2}\right)^{2}+\frac{1}{4}(1-p) \hat{a}-\frac{1}{2}(1-2 p) \hat{b}\right) \\
+ & z^{2}\left(\left(6 \hat{a}^{2}-8 \hat{c}\right)(\theta+1)^{4}+\left(\frac{3}{2}(5+2 p) \hat{a}^{2}-4 \hat{a} \hat{b}-2(13+2 p) \hat{c}\right)(\theta+1)^{2}\right. \\
& \left.+\frac{3}{4} \hat{a}^{2}-(1-p) \hat{a} \hat{b}+\hat{b}^{2}-\left(2+2 p-p^{2}\right) \hat{c}\right) \\
& -\left(\hat{a}^{2}-4 \hat{c}\right) z^{3}\left(\theta+\frac{3}{2}\right)^{2}\left(4 \hat{a}\left(\theta+\frac{3}{2}\right)^{2}+3(1+p) \hat{a}-2 \hat{b}\right) \\
+ & \left(\hat{a}^{2}-4 \hat{c}\right)^{2} z^{4}\left(\theta+\frac{3}{2}\right)\left(\theta+\frac{5}{2}\right)\left(\theta+\frac{3}{2}+\alpha\right)\left(\theta+\frac{5}{2}-\alpha\right),
\end{aligned}
$$

where $p=\alpha(1-\alpha)$.

Theorem 5.5. Let $\hat{F}(z) \in 1+z \mathbb{C} \llbracket z \rrbracket$ be the analytic solution of the differential equation $D y=0$ with $D$ defined in (5.21), and let $F(z) \in 1+z \mathbb{C} \llbracket z \rrbracket$ be the Hadamard product of

$$
f_{\alpha}(z)=\frac{1}{1-z} \cdot{ }_{2} F_{1}\left(\begin{array}{c|c}
\alpha, 1-\alpha & \frac{-z}{1-z}
\end{array}\right)
$$

and the analytic solution of the differential equation with differential operator (2.5). Then

$$
F(z)=\frac{1-c z^{2}}{\left(1-a z+c z^{2}\right)^{3 / 2}} \hat{F}\left(\frac{-z}{1-a z+c z^{2}}\right) .
$$

Proof. As before, the proof is just an extensive check using MAPLE; the differential equation for the Hadamard product $F(z)$ is too lengthy to be given here.

We remark that Theorem 4.1 in $\S 4$ may be regarded as a limiting case $\alpha \rightarrow 0$ of Theorem 5.5. 
Table 1. Equivalences relating the sporadic cases (4.8) and (4.12)

\begin{tabular}{ccccc}
\hline & $(\mathrm{A})$ & $(\mathrm{B})$ & $(\mathrm{C})$ & $(\mathrm{D})$ \\
\hline$(\delta)$ & $(\mathrm{e}) *(\mathrm{a})$ & $(\mathrm{h}) *(\mathrm{a})$ & $(\mathrm{i}) *(\mathrm{a})$ & $(\mathrm{j}) *(\mathrm{a})$ \\
$(\eta)$ & $(\mathrm{e}) *(\mathrm{~b})$ & $(\mathrm{h}) *(\mathrm{~b})$ & $(\mathrm{i}) *(\mathrm{~b})$ & $(\mathrm{j}) *(\mathrm{~b})$ \\
$(\alpha)$ & $(\mathrm{e}) *(\mathrm{c})$ & $(\mathrm{h}) *(\mathrm{c})$ & $(\mathrm{i}) *(\mathrm{c})$ & $(\mathrm{j}) *(\mathrm{c})$ \\
$(\epsilon)$ & $(\mathrm{e}) *(\mathrm{~d})$ & $(\mathrm{h}) *(\mathrm{~d})$ & $(\mathrm{i}) *(\mathrm{~d})$ & $(\mathrm{j}) *(\mathrm{~d})$ \\
$(\zeta)$ & $(\mathrm{e}) *(\mathrm{f})$ & $(\mathrm{h}) *(\mathrm{f})$ & $(\mathrm{i}) *(\mathrm{f})$ & $(\mathrm{j}) *(\mathrm{f})$ \\
$(\gamma)$ & $(\mathrm{e}) *(\mathrm{~g})$ & $(\mathrm{h}) *(\mathrm{~g})$ & $(\mathrm{i}) *(\mathrm{~g})$ & $(\mathrm{j}) *(\mathrm{~g})$ \\
\hline
\end{tabular}

Note that Theorems 5.1 and 5.3 are special cases of Theorem 5.5, but in the former cases we can explicitly write down the Hadamard products involved, through hypergeometric series. Theorem 5.5 provides us with equivalences relating the sporadic cases (4.8) and (4.12), namely, it gives us the list of 24 equivalences in Table 1.

Theorem 5.1 gives in a nice way the equivalence of the YY-pullbacks of the fifth-order hypergeometric on the one side and Hadamard products of two second-order Legendrian cases on the other side, while Theorem 5.3 provides the equivalence of $(\mathrm{X}) *(\mathrm{x})$ and the YY-pullback of $(\mathrm{X}) *(\xi)$, where $(\mathrm{X})$ is one of the hypergeometric cases $(4.1),(4.2),(\mathrm{x})$ is one of the second-order Legendrian equations (4.5), (4.6), and $(\xi)$ is the corresponding third-order Legendrian equation (4.10), (4.11).

We have already established the algebraic connection between the YY-pullback of the left-hand side in (5.15) and (e)*(e) (Theorem 5.1); in (5.15) we have the equivalence implying, in particular, the equivalence of the YY-pullback of $(\mathrm{C}) *(\vartheta)$ and of $(\mathrm{e}) *(\mathrm{e})$. Finally, the equivalence of the YY-pullback of $(\mathrm{C}) *(\vartheta)$ and of $(\mathrm{C}) *(\mathrm{i})$ follows from Theorem 5.3; this implies the equivalence of $(\mathrm{e}) *(\mathrm{e})$ and $(\mathrm{C}) *(\mathrm{i})$, which is also the subject of Theorem 5.4.

We now illustrate Theorem 5.5 by an explicit example of an algebraic transformation relating two Calabi-Yau equations.

Example 5.6. Let us write the algebraic transformation for the equivalence of (the YY-pullback of $)(\mathrm{C}) *(\gamma)$ and $(\mathrm{i}) *(\mathrm{~g})$.

We have the following solutions of the fifth-order equation for $(\mathrm{C}) *(\gamma)$ :

$$
\begin{aligned}
& w_{0}(z)=\sum_{n=0}^{\infty} z^{n} \frac{(4 n) !}{n !^{2}(2 n) !} \sum_{k=0}^{n}\left(\begin{array}{c}
n \\
k
\end{array}\right)^{2}\left(\begin{array}{c}
n+k \\
n
\end{array}\right)^{2}, \\
& w_{1}(z)=w_{0}(z) \log z+\sum_{n=1}^{\infty} z^{n} \frac{(4 n) !}{n !^{2}(2 n) !} \sum_{k=0}^{n}\left(\begin{array}{c}
n \\
k
\end{array}\right)^{2}\left(\begin{array}{c}
n+k \\
n
\end{array}\right)^{2} \\
& \times\left(4 H_{4 n}-2 H_{2 n}-2 H_{n}-2 H_{n-k}+2 H_{n+k}\right),
\end{aligned}
$$

with the YY-pullback

$$
\hat{F}(z)=\left(1-2176 z+4096 z^{2}\right)^{-1 / 2}\left(w_{0}(z) \cdot \theta w_{1}(z)-\theta w_{0}(z) \cdot w_{1}(z)\right)^{1 / 2},
$$

where we used the data $C_{\alpha}=64$ and $\hat{a}=17, \hat{c}=1$ for cases $(\mathrm{C})$ and $(\gamma)$. 
For (i) and (g) we have $C_{\alpha}=64$ and $a=17, c=72$, and the analytic solution is

$$
F(z)=\sum_{n=0}^{\infty} z^{n} \sum_{0 \leqslant j \leqslant i \leqslant n}(-1)^{i} 8^{n-i}\left(\begin{array}{l}
n \\
i
\end{array}\right)\left(\begin{array}{l}
i \\
j
\end{array}\right)^{3} \sum_{k=0}^{n}(-1)^{k}\left(\begin{array}{l}
n \\
k
\end{array}\right) \frac{(4 k) !}{k !^{2}(2 k) !} .
$$

Then Theorem 5.5 gives us the transformation

$$
F(z)=\frac{1-294912 z^{2}}{\left(1-1088 z+294912 z^{2}\right)^{3 / 2}} \cdot \hat{F}\left(\frac{-z}{1-1088 z+294912 z^{2}}\right),
$$

which we can write in a form resembling Clausen's Formula:

$$
\begin{aligned}
&\left(\sum_{n=0}^{\infty} z^{n} \sum_{0 \leqslant j \leqslant i \leqslant n}(-1)^{i} 8^{n-i}\left(\begin{array}{l}
n \\
i
\end{array}\right)\left(\begin{array}{l}
i \\
j
\end{array}\right)^{3} \sum_{k=0}^{n}(-1)^{k}\left(\begin{array}{l}
n \\
k
\end{array}\right) \frac{(4 k) !}{k !^{2}(2 k) !}\right)^{2} \\
&=\frac{1}{1-} \frac{1088 z+294912 z^{2}}{\sum_{n=0}^{\infty}\left(\frac{-z}{1-1088 z+294912 z^{2}}\right)^{n}} \\
& \times \sum_{k=0}^{n} \frac{(4 k) !}{k !^{2}(2 k) !} \frac{(4 n-4 k) !}{(n-k) !^{2}(2 n-2 k) !} \sum_{j=0}^{n-k}\left(\begin{array}{c}
n-k \\
j
\end{array}\right)^{2}\left(\begin{array}{c}
n-k+j \\
n-k
\end{array}\right)^{2} \\
& \times \sum_{l=0}^{k}\left(\begin{array}{c}
k \\
l
\end{array}\right)^{2}\left(\begin{array}{c}
k+l \\
k
\end{array}\right)^{2}\left(1+(2 k-n)\left(4 H_{4 k}-2 H_{2 k}-2 H_{k}-2 H_{k-l}+2 H_{k+l}\right)\right) .
\end{aligned}
$$

Theorem 5.7. Let $F(z)$ be the Hadamard square of the series $f_{\alpha}(z)$ given in (5.2), while $\tilde{F}(z)$ is the Hadamard product of

$$
(1-4 z)^{-1 / 2}=\sum_{n=0}^{\infty}\left(\begin{array}{c}
2 n \\
n
\end{array}\right) z^{n} \quad \text { and } \quad g_{\alpha}(z)
$$

in (5.9), (5.12). Then

$$
F(z)=\frac{1}{1+z} \tilde{F}\left(\frac{z}{(1+z)^{2}}\right) .
$$

As before, Calabi-Yau applications of Theorem 5.7 correspond to the choices $\alpha \in$ $\left\{\frac{1}{2}, \frac{1}{3}, \frac{1}{4}, \frac{1}{6}\right\}$.

Finally, we present more algebraic transformations of fourth-order Calabi-Yau differential equations which are not covered by the above theorems but look quite nice (to our taste).

Theorem 5.8. The following identities are valid:

$$
\begin{aligned}
& \sum_{n=0}^{\infty}\left(\begin{array}{c}
2 n \\
n
\end{array}\right) \sum_{k, l}(-1)^{k+l}\left(\begin{array}{l}
n \\
k
\end{array}\right)\left(\begin{array}{c}
n \\
l
\end{array}\right)\left(\begin{array}{c}
k+l \\
k
\end{array}\right)^{3} z^{n} \\
& =\frac{1}{\sqrt{1-4 z}} \sum_{n=0}^{\infty}\left(\begin{array}{c}
2 n \\
n
\end{array}\right) \sum_{k=0}^{n}\left(\begin{array}{l}
n \\
k
\end{array}\right)^{2}\left(\begin{array}{c}
n+k \\
n
\end{array}\right)\left(\begin{array}{c}
3 k \\
n
\end{array}\right)\left(\frac{z}{1-4 z}\right)^{n},
\end{aligned}
$$




$$
\begin{aligned}
& \sum_{n=0}^{\infty}\left(\begin{array}{c}
2 n \\
n
\end{array}\right) \sum_{k=0}^{n}\left(\begin{array}{l}
n \\
k
\end{array}\right)^{2}\left(\begin{array}{c}
2 k \\
k
\end{array}\right)\left(\begin{array}{c}
2 n-2 k \\
n-k
\end{array}\right) z^{n} \\
& \quad=\frac{1}{\sqrt{1-32 z}} \sum_{n=0}^{\infty}\left(\begin{array}{c}
2 n \\
n
\end{array}\right) \sum_{k, l}(-1)^{n-k} 2^{3(n-k)}\left(\begin{array}{l}
n \\
k
\end{array}\right)\left(\begin{array}{c}
k \\
l
\end{array}\right)^{2}\left(\begin{array}{c}
2 l \\
l
\end{array}\right)\left(\begin{array}{c}
2 k-2 l \\
k-l
\end{array}\right)\left(\frac{z}{1-32 z}\right)^{n},
\end{aligned}
$$

$$
\begin{gathered}
\sum_{n=0}^{\infty}\left(\begin{array}{c}
2 n \\
n
\end{array}\right) \sum_{k, l}\left(\begin{array}{l}
n \\
k
\end{array}\right)\left(\begin{array}{c}
n \\
l
\end{array}\right)\left(\begin{array}{c}
k \\
l
\end{array}\right)\left(\begin{array}{c}
k+l \\
k
\end{array}\right)\left(\begin{array}{c}
2 l \\
l
\end{array}\right)\left(\begin{array}{c}
2 k \\
k-l
\end{array}\right) z^{n} \\
=\frac{1}{\sqrt{1-4 z}} \sum_{n=0}^{\infty}\left(\begin{array}{c}
2 n \\
n
\end{array}\right)^{2} \sum_{k=0}^{n}\left(\begin{array}{l}
n \\
k
\end{array}\right)^{2}\left(\begin{array}{c}
2 k \\
k
\end{array}\right)\left(\frac{z}{1-4 z}\right)^{n}
\end{gathered}
$$

$$
\begin{aligned}
& \sum_{n=0}^{\infty}\left(\sum_{k=0}^{n}\left(\begin{array}{l}
n \\
k
\end{array}\right)^{2}\left(\begin{array}{c}
n+k \\
n
\end{array}\right)\right)^{2} z^{n} \\
& \quad=\frac{1}{1+z} \sum_{n=0}^{\infty}\left(\begin{array}{c}
2 n \\
n
\end{array}\right) \sum_{k, l}\left(\begin{array}{l}
n \\
k
\end{array}\right)\left(\begin{array}{c}
n \\
l
\end{array}\right)\left(\begin{array}{c}
k+l \\
k
\end{array}\right)\left(\begin{array}{c}
2 l \\
l
\end{array}\right)\left(\begin{array}{c}
l \\
k-l
\end{array}\right)\left(\frac{z}{(1+z)^{2}}\right)^{n},
\end{aligned}
$$

$$
\begin{aligned}
& \sum_{n=0}^{\infty}\left(\sum_{k=0}^{n}\left(\begin{array}{c}
n \\
k
\end{array}\right)\left(\begin{array}{c}
2 k \\
k
\end{array}\right)\left(\begin{array}{c}
2 n-2 k \\
n-k
\end{array}\right)\right)^{2} z^{n} \\
& \quad=\frac{1}{1-32 z} \sum_{n=0}^{\infty}\left(\begin{array}{c}
2 n \\
n
\end{array}\right) \sum_{k=0}^{[n / 2]} 2^{n-2 k}\left(\begin{array}{c}
n \\
k
\end{array}\right)\left(\begin{array}{c}
n-k \\
k
\end{array}\right)\left(\begin{array}{c}
2 k \\
k
\end{array}\right)\left(\begin{array}{c}
2 n-2 k \\
n-k
\end{array}\right)\left(\frac{z}{(1-32 z)^{2}}\right)^{n},
\end{aligned}
$$

$$
\begin{aligned}
\sum_{n=0}^{\infty}\left(\sum_{k=0}^{[n / 3]}(-1)^{k} 3^{n-3 k}\left(\begin{array}{c}
n \\
3 k
\end{array}\right) \frac{(3 k) !}{k !^{3}}\right)^{2} z^{n} \\
=\frac{1}{1-27 z} \sum_{n=0}^{\infty} \sum_{k=0}^{[n / 3]}(-1)^{n-k}\left(\left(\begin{array}{c}
2 n-3 k-1 \\
n
\end{array}\right)+\left(\begin{array}{c}
2 n-3 k \\
n
\end{array}\right)\right) \\
\quad \times \frac{(3 k) !}{k !^{3}} \frac{(3 n-3 k) !}{(n-k) !^{3}}\left(\frac{z}{(1-27 z)^{2}}\right)^{n}
\end{aligned}
$$

\section{The invariance of Yukawa couplings}

As we have already seen, algebraic transformations transform Calabi-Yau equations into similar ones, but sometimes look quite different. Such transformations, however, preserve (in a certain precise sense, which we describe below) the Yukawa coupling of the corresponding differential equations. Recall that the Yukawa coupling $K$ can be defined, up to a normalization constant factor, through the quotient $t(z)=y_{1}(z) / y_{0}(z) \in \log z+z \mathbb{Q} \llbracket z \rrbracket$ of the two solutions $y_{0}(z) \in 1+z \mathbb{Z} \llbracket z \rrbracket$ and $y_{1}(z)$ of a Calabi-Yau equation (3.3) (see $\S 2$ ) 
as

$$
K=\frac{1}{y_{0}^{2} \cdot(\mathrm{d} t / \mathrm{d} z)^{3}} \exp \left(-\frac{1}{2} \int^{z} P(z) \mathrm{d} z\right)
$$

and this function is often viewed as a function of $q=\mathrm{e}^{t}$, since its $q$-expansion in the case of a degenerating family of Calabi-Yau 3-folds is supposed to encode the counting of rational curves of various degrees on a mirror manifold.

On the other hand, we did find several examples of Calabi-Yau equations whose Yukawa couplings coincide, although it is not obvious that the equations themselves are indeed equivalent in the sense that they are related by an algebraic transformation. At the time of writing, we have discovered and proved algebraic transformations for all pairs of Calabi-Yau equations with equal Yukawa couplings tabulated in [3] (see also the diploma thesis [9]). Most of these transformations (at least those that follow a general pattern) were given in $\S 5$ and some of them are immediate consequences of the theorems proven therein. Section 7 describes some of our strategies for finding the transformations.

It is routine to write down the fourth-order linear differential equation

$$
\frac{\mathrm{d}^{4} Y}{\mathrm{~d} x^{4}}+\hat{P} \frac{\mathrm{d}^{3} Y}{\mathrm{~d} x^{3}}+\hat{Q} \frac{\mathrm{d}^{2} Y}{\mathrm{~d} x^{2}}+\hat{R} \frac{\mathrm{d} Y}{\mathrm{~d} x}+\hat{S} Y=0
$$

for the function $Y(x)=v(x) \cdot y(z(x))$. For example, we have

$$
\hat{P}=-6 \frac{z^{\prime \prime}}{z^{\prime}}+z^{\prime} P-4 \frac{v^{\prime}}{v}
$$

where the prime denotes the $x$-derivative.

Clearly, the new equation (6.2) does not necessarily have rational coefficients, but it does after we impose certain conditions on $v(x)$ and $z(x)$ (for instance, assuming their rationality). Continuing the computation in (6.3) we obtain the following.

Proposition 6.1 (cf. [12]). Define

$$
U_{z}(P, Q)=Q-\frac{3}{2} \frac{\mathrm{d} P}{\mathrm{~d} z}-\frac{3}{8} P^{2} .
$$

Then

$$
U_{x}(\hat{P}, \hat{Q})-\left(z^{\prime}\right)^{2} U_{z}(P, Q)=5\{z, x\}
$$

where

$$
\{z, x\}=\frac{z^{\prime \prime \prime}}{z^{\prime}}-\frac{3}{2}\left(\frac{z^{\prime \prime}}{z^{\prime}}\right)^{2}
$$

is the Schwarzian derivative.

Our next statement shows the invariance of the Yukawa coupling.

Proposition 6.2. Let $Y(x)=v(x) \cdot y(z(x))$, where $z(x)=x+O\left(x^{2}\right)$ and $v(x)=$ $1+O(x)$. Then the Yukawa couplings defined in accordance with (6.1) coincide:

$$
K_{Y(x)}=K_{y(z)} .
$$


Proof. Because both the mirror map $t(z)$ and the Yukawa coupling depend on quotients of the solutions rather than on the solutions themselves, it is sufficient to treat the case $v(x)=1$. We have the formula (6.1) implying

$$
K=\frac{y_{0}^{4}}{\operatorname{det}\left(\begin{array}{cc}
y_{0} & y_{1} \\
\mathrm{~d} y_{0} / \mathrm{d} z & \mathrm{~d} y_{1} / \mathrm{d} z
\end{array}\right)^{3}} \exp \left(-\frac{1}{2} \int^{z} P(z) \mathrm{d} z\right)
$$

Furthermore,

$$
\frac{\mathrm{d} Y_{0}}{\mathrm{~d} x}=z^{\prime} \frac{\mathrm{d} y_{0}}{\mathrm{~d} z}, \quad \frac{\mathrm{d} Y_{1}}{\mathrm{~d} x}=z^{\prime} \frac{\mathrm{d} y_{1}}{\mathrm{~d} z} \quad \text { and } \quad \tilde{P}=-6 \frac{z^{\prime \prime}}{z^{\prime}}+z^{\prime} P
$$

hence,

$$
\begin{aligned}
K_{Y(x)}= & \frac{Y_{0}^{4}}{\operatorname{det}\left(\begin{array}{cc}
Y_{0} & Y_{1} \\
\mathrm{~d} Y_{0} / \mathrm{d} x & \mathrm{~d} Y_{1} / \mathrm{d} x
\end{array}\right)^{3}} \exp \left(-\frac{1}{2} \int^{x} \tilde{P}(x) \mathrm{d} x\right) \\
= & \frac{y_{0}(z)^{4}}{\operatorname{det}\left(\begin{array}{cc}
y_{0}(z) & y_{1}(z) \\
z^{\prime} \mathrm{d} y_{0} / \mathrm{d} z & z^{\prime} \mathrm{d} y_{1} / \mathrm{d} z
\end{array}\right)^{3}} \exp \left(-\frac{1}{2} \int^{x}\left(-6 \frac{z^{\prime \prime}}{z^{\prime}}+z^{\prime} P(z(x))\right) \mathrm{d} x\right) \\
= & \frac{y_{0}(z)^{4}}{\operatorname{det}\left(\begin{array}{cc}
y_{0}(z) & y_{1}(z) \\
\mathrm{d} y_{0} / \mathrm{d} z & \mathrm{~d} y_{1} / \mathrm{d} z
\end{array}\right)^{3}} \exp \left(-\frac{1}{2} \int^{z} P(z) \mathrm{d} z\right) \\
= & K_{y(z)} .
\end{aligned}
$$

Here we used $z^{\prime}(0)=1$ when we integrated

$$
3 \int_{0}^{x} \frac{z^{\prime \prime}}{z^{\prime}} \mathrm{d} x=3 \log z^{\prime}(x)-3 \log z^{\prime}(0) .
$$

From the transformation formulae for passing from (3.3) to (6.2) through the map $Y(x)=v(x) \cdot y(z(x))$, we find that the Calabi-Yau condition (3.4) is preserved. This is very hard to see by direct computation, since one gets an enormous fourth-order nonlinear differential equation for $z(x)$.

Conjecture 6.3. If Yukawa couplings coincide, then there exists an algebraic transformation between corresponding Calabi-Yau differential equations.

In fact, Proposition 6.2 states that the Yukawa coupling defined by (6.1) is preserved by any formal coordinate transformation $z(x)=x+\cdots$. However, the requirement for the transformed equation to be of Calabi-Yau type (in particular, to have rational functions as coefficients) should lead to the algebraicity of such a transformation. 


\section{Proof of Theorem 5.4: guessing algebraic transformations}

Not only does this section provide a proof of Theorem 5.4 but we also illustrate our strategies to guess algebraic transformations for Calabi-Yau differential equations on the example of Theorem 5.4; more precisely, we show how to 'discover' the equivalence of $(\mathrm{e}) *(\mathrm{e})$ and $(\mathrm{C}) *(\mathrm{i})$. We distinguish three methods: two analytic and one algebraic. They also provide proofs of the discovered algebraic transformation as soon as the fact of its existence is established.

First of all we indicate a way to recognize in MAPLE whether a function $R(z)$, given by its Taylor expansion at the origin, is rational or algebraic and, if it is, to find a closed expression. For this, one applies seriestodiffeq $(R, \hat{R}(z))$ (with gfun) and then dsolve to $\hat{R}(z)$.

\subsection{Analytic guessing}

Compute the $z$-expansions (30 terms, say) of the mirror maps $\tilde{q}$ and $q$, then write

$$
\tilde{q}(z)= \pm q\left( \pm z+a_{2} z^{2}+\cdots+a_{30} z^{30}+\cdots\right)
$$

(the signs belong together); expand the latter equality up to $z^{31}$ to get a system of linear equations for unknowns $a_{2}, \ldots, a_{30}$. It takes MAPLE a few minutes to solve the system; this finds the inner transformation. Then compute the power series expansion of the outer transformation multiple and use gfun.

\subsection{Schwarzian relation}

In passing from $(\mathrm{e}) *(\mathrm{e})$ to $(\mathrm{C}) *(\mathrm{i})$, we can use the 'magic Schwarzian relation' (Proposition 6.1) for $z(x)$ in the form $z(x)=-x+\cdots$. Then we recursively find the expansion for $\tilde{z}(x)=-256 z(x / 256)$,

$$
\tilde{z}(x)=x+10 x^{2}+83 x^{3}+628 x^{4}+4501 x^{5}+31134 x^{6}+210023 x^{7}+\cdots,
$$

which MAPLE easily recognizes as the expansion of a rational function

$$
\tilde{z}(x)=\frac{x(1-x)^{2}}{\left(1-6 x+x^{2}\right)^{2}} .
$$

Furthermore, denoting by $Y(z), \hat{Y}(z) \in 1+z \mathbb{Z} \llbracket z \rrbracket$ the analytic solutions of $(\mathrm{e}) *(\mathrm{e})$ and $(\mathrm{C}) *(\mathrm{i})$, respectively, it remains to let MAPLE identify the quotient of

$$
Y(z) \quad \text { and } \quad \hat{Y}\left(\frac{-z(1-256 z)^{2}}{\left(1-6 \cdot 256 z+256^{2} z^{2}\right)^{2}}\right)
$$

with the algebraic function $\left(1-6 \cdot 256 z+256^{2} z^{2}\right)^{-1 / 2}$. 


\subsection{Local monodromy considerations}

The operator for the Hadamard product $(\mathrm{e}) *(\mathrm{e})$ is

$$
D=\theta^{4}-16 z\left(16 \theta^{4}+128 \theta^{3}+112 \theta^{2}+48 \theta+9\right)+\cdots-2^{40} z^{5}(\theta+1)^{4} .
$$

The discriminant is $(1+256 z)^{2}(1-256 z)^{3}$ but the point $z=-\frac{1}{256}$ turns out to be an apparent singularity. The point $z=\frac{1}{256}$ is also a MUM point and a calculation shows that

$$
K(q)_{z=1 / 256}=K\left(q^{2}\right)_{z=0}=K\left(q^{2}\right)_{z=\infty} .
$$

Thus, the operator $D$ has three MUM points and no other singularities.

The operator corresponding to the Hadamard product $(\mathrm{C}) *(\mathrm{i})$ is

$$
\theta^{4}-16 z(4 \theta+1)(4 \theta+3)\left(32 \theta^{2}+32 \theta+13\right)+2^{16} z^{2}(4 \theta+1)(4 \theta+3)(4 \theta+5)(4 \theta+7),
$$

an operator with discriminant $(1-4096 z)^{2}$. The exponents at $z=\frac{1}{4096}$ are $0, \frac{1}{4}, \frac{3}{4}, 1$; at $z=\infty$ they are $\frac{1}{4}, \frac{3}{4}, \frac{5}{4}, \frac{7}{4}$, hence these points have local monodromy of order 4 . All these facts are easily checked with MAPLE using formal_sol (within DEtools). If we try to think of $(\mathrm{e}) *(\mathrm{e})$ as a pullback of $(\mathrm{C}) *(\mathrm{i})$ via a rational map $R(z)=P(z) / Q(z)$, we see that it requires the following properties: $R^{-1}(0)=\left\{0, \frac{1}{256}, \infty\right\}$ and the ramification over $z=\frac{1}{256}$ is of order 2. Therefore, the degree of $R(z)$ is four. We also require $R^{-1}\left(\frac{1}{4096}\right)=\left\{-\frac{1}{256}\right\}$ as we want the ramification index at $z=\frac{1}{256}$ to be 4 . But then we cannot require the same ramification index over $z=\infty$; hence, we assume that $R^{-1}(\infty)$ consists of two points, each with ramification index 2 . The pullback then has exponents $\frac{1}{2}$ at these points, and we have to divide by the square root of the polynomial defining these points. Combining the information we see that

$$
R(z)=c \frac{z(1-256 z)^{2}}{q(z)^{2}}, \quad q(z)=1+a z+b z^{2},
$$

where $a, b$ and $c$ are certain constants. We now determine the constants by requiring that $R\left(-\frac{1}{256}\right)=\frac{1}{4096}$ and

$$
R\left(x-\frac{1}{256}\right)=\frac{1}{4096}+0 \cdot x+0 \cdot x^{2}+0 \cdot x^{3}+\cdots .
$$

We find

$$
a=-1536, \quad b=65536, \quad c=-1 .
$$

If $\hat{Y}(z)$ is the solution of $(\mathrm{C}) *(\mathrm{i})$, then the function $\hat{Y}(R(z))$ has the square-root behaviour at the pre-image of $z=\infty$, that is, at the roots of $q(z)=1-1536 z+65536 z^{2}$; hence,

$$
Y(z)=\frac{1}{\sqrt{q(z)}} \hat{Y}\left(\frac{-z(1-256 z)^{2}}{q(z)^{2}}\right)
$$

has the same local properties as the solution of $(\mathrm{e}) *(\mathrm{e})$ and, in fact, coincides with it.

The same method can be used to find the transformation in the other cases. For example, from the fact that the Hadamard product multiplies the singular points of the 
operators, it follows without further calculation that the operators (5.20) and (5.21) have $0, \infty$ and the roots of $1-2 \hat{a} z+\hat{c} z^{2}$ as singularities. The Hadamard product of $f_{\alpha}(z)$ and operator (2.5) has its singularities at $0, \infty$ and the roots of $1-a z+c z^{2}$. A possible transformation of degree 2 will have to map these singular points in exactly the same way as in Theorem 4.1; hence, we are again led to consider $z \mapsto-z /\left(1-a z+c z^{2}\right)$. In this case, the prefactor can also be determined by looking at the local exponents.

\section{Concluding remarks}

It is definitely not our goal here to stress the consequences of our theorems, since we feel that the transformations, and even their existence, are beautiful by themselves. Our results provide (albeit rather indirect) geometric interpretations of several (YY-) pullbacks from the table in [3]; previously, such pullbacks were of geometric origin only conjecturally, and no relation to Calabi-Yau geometry was known. Another application of our transformation theorems, having a more arithmetic flavour, is the integrality of the analytic solutions of the pullbacks (condition (ii) of $\S 2$ ), as well as the integrality of the corresponding mirror maps (condition (iii)) when the results in [11] are applicable. There are many aspects that can be discussed elsewhere.

Acknowledgements. The work of G.A. and W.Z. was supported by the Max Planck Institute for Mathematics (Bonn). W.Z. acknowledges support by the Hausdorff Center for Mathematics (Bonn). We thank Fernando Rodríguez Villegas and Don Zagier for pointing out the way to make our Theorem 5.5 as general as it is now. Special thanks go to Michael Bogner and Stefan Reiter for many useful remarks and their discovery of the 'strange' Calabi-Yau differential equation corresponding to (2.7). Finally, we thank the referee for many helpful suggestions.

The foundations of this work were established during a visit of W.Z. to the Institute of Algebraic Meditation at Höör, Sweden (May 26-June 3, 2007). He thanks the staff of the institute for their hospitality.

\section{References}

1. G. Almkvist, Calabi-Yau differential equations of degree 2 and 3 and Yifan Yang's pullback, Preprint (http://arxiv.org/abs/math.AG/0612215; 2006).

2. G. Almkvist and W. Zudilin, Differential equations, mirror maps and zeta values, in Mirror symmetry V (ed. N. Yui, S.-T. Yau and J. D. Lewis), AMS/IP Studies in Advanced Mathematics, Volume 38, pp. 481-515 (American Mathematical Society and International Press, Providence, RI, 2006).

3. G. Almkvist, C. van Enckevort, D. van Straten and W. Zudilin, Tables of CalabiYau equations, Preprint (http://arxiv.org/abs/math.AG/0507430; 2005).

4. Y. ANDRÉ, G-functions and geometry, Aspects of Mathematics, Volume 13 (Vieweg \& Sohn, Braunschweig, 1989).

5. R. ApÉRY, Irrationalité de $\zeta(2)$ et $\zeta(3)$, in Journées arithmétiques de Luminy, Astérisque, Volume 61, pp. 11-13 (Société Mathématique de France, Paris, 1979).

6. A. Beauville, Les familles stables de courbes elliptiques sur $\mathbb{P}^{1}$ admettant quatre fibres singulières, C. R. Acad. Sci. Paris Sér. I 294 (1982), 657-660. 
7. F. BEukers, Irrationality of $\pi^{2}$, periods of an elliptic curve and $\Gamma_{1}(5)$, in Diophantine approximations and transcendental numbers, Progress in Mathematics, Volume 31, pp. 4766 (Birkhäuser, 1983).

8. F. Beukers, On Dwork's accessory parameter problem, Math. Z. 241 (2002), 425-444.

9. M. Bogner, Differentielle Galoisgruppen und Transformationstheorie für Calabi-Yau-Operatoren vierter Ordnung, Diploma-Thesis, Institut für Mathematik, Johannes GutenbergUniversität, Mainz (2008).

10. V. V. Golyshev, Classification problems and mirror duality, in Surveys in geometry and number theory: reports on contemporary Russian mathematics, London Mathematical Society Lecture Note Series, Volume 338, pp. 88-121 (Cambridge University Press, 2007).

11. C. Krattenthaler and T. Rivoal, Multivariate $p$-adic formal congruences and integrality of Taylor coefficients of mirror maps, in Théories galoisiennes et arithmétiques des équations différentielles (ed. L. Di Vizio and T. Rivoal), Séminaires et Congrès (Société Mathématique de France, Paris (in press).

12. B. H. LIAN AND S.-T. YAU, Differential equations from mirror symmetry, in Surveys in differential geometry: differential geometry inspired by string theory, Surveys in Differential Geometry, Volume 5, pp. 510-526 (International Press, Boston, MA, 1999).

13. R. Miranda And U. Persson, On extremal rational elliptic surfaces, Math. Z. 193 (1986), 537-558.

14. C. Peters, Monodromy and Picard-Fuchs equations for families of $K 3$-surfaces and elliptic curves, Annales Sci. École Norm. Sup. (4) 19 (1986), 583-607.

15. C. Peters and J. Stienstra, A pencil of $K 3$-surfaces related to Apéry's recurrence for $\zeta(3)$ and Fermi surfaces for potential zero, in Arithmetic of complex manifolds, Lecture Notes in Mathematics, Volume 1399, pp. 110-127 (Springer, 1989).

16. U. Schmickler-Hirzebruch, Elliptische Flächen über $\mathbb{P}^{1} \mathbb{C}$ mit drei Ausnahmefasern und die hypergeometrische Differentialgleichung, Schriftenreihe des Mathematischen Instituts der Universität Münster, 2. Serie 33 (Universität Münster, Mathematisches Institut, Münster, 1985).

17. L. J. Slater, Generalized hypergeometric functions (Cambridge University Press, 1966).

18. C. VAN ENCKEVORT AND D. VAN StRAten, Monodromy calculations of fourth-order equations of Calabi-Yau type, in Mirror symmetry $V$ (ed. N. Yui, S.-T. Yau and J. D. Lewis), AMS/IP Studies in Advanced Mathematics, Volume 38, pp. 539-559 (American Mathematical Society and International Press, Providence, RI, 2006).

19. E. T. Whittaker And G. N. Watson, A course of modern analysis, 4 th edn (Cambridge University Press, 1927).

20. D. ZAGIER, Integral solutions of Apéry-like recurrence equations, in Groups and symmetries: from Neolithic Scots to John McKay, CRM Proceedings and Lecture Notes, Volume 47, pp. 349-366 (American Mathematical Society, Providence, RI, 2009).

21. W. Zudilin, Quadratic transformations and Guillera's formulas for $1 / \pi^{2}$, Math. Notes 81 (2007), 297-301. 